Research

\title{
Prevalence and determinants of dietary supplement and non-prescription medicine use by men and women over 53 years old in Taiwan. Results from a population-based cross-sectional survey
}

\author{
Alan C Tsai \\ Human Nutrition Program, Department of Environmental Health \\ Sciences, School of Public Health, University of Michigan, Ann Arbor, \\ Michigan, USA
}

Jenn-Chang Liou

Department of Applied Health Science, Asia University, Wu-feng, Taichung, Taiwan

Jack M C Chang

Department of Health Management and Administration, Asia University, Wu-feng, Taichung, Taiwan

\section{Yi-Li Chuang}

Population and Health Research Center, Bureau of Health Promotion, Department of Health, Taichung, Taiwan

\section{Shu-Hui Lin and Yu-Hsuan Lin}

Bureau of Health Promotion, Department of Health, Taichung, Taiwan

Objectives: This study investigated the prevalence and determinants of the use of dietary supplements and non-prescription medicines by older adults in Taiwan.

Methods: Data are from the 1999 Survey of Health and Living Status of the Elderly in Taiwan. The survey involved a national random sample of 2360 men and 2080 women, 53 years or older.

Results: Overall, $32.4 \%$ of elderly men and $42.7 \%$ of elderly women used at least one kind of dietary supplement during the past 12 months. Approximately $16 \%$ of elderly men and $19 \%$ of elderly women used multivitamin and mineral supplements, 10.5 and $22 \%$ used calcium, and 10 and $14 \%$ used vitamin $E$, respectively.

Conclusions: The rates of supplement use are generally lower than those observed in the USA but comparable to that in other industrialised countries. Elderly who are females or who are more highly educated or physically more active are more frequent supplement users.

Key words: dietary supplements, elderly, non-prescription medicine, Taiwan.

Correspondence to: Dr Alan C. Tsai, School of Public Health, University of Michigan.Email: atsai@umich.edu

\section{Introduction}

The use of dietary supplements including vitamins and minerals and other nutritional products (non-vitamin and non-mineral supplements) has been popular in the USA and many other industrialised countries in recent years [1-7]. The prevalence of use of these products is less well understood in many other parts of the world. Knowledge of the population's supplement use is important for both nutrition research and health-care policy-making. The use of dietary supplements may alter one's nutritional status by contributing significantly to an individual's nutrient intake, especially micronutrients [8,9]. Some supplements such as antioxidant vitamins may also help to minimise oxidative damage to tissues, reducing the risk of chronic diseases $[10,11]$. However, it is also important to monitor potential inadequate or excessive intake in the population. In addition to vitamin/mineral supplements, many individuals also practise self-medication with herbs, herbal products or other nonprescription medicines for their health problems [12]. Such a practice could increase the risk of drug-drug or drug-nutrient interactions, delay proper treatment or mask the development of diseases [13-15]. Self-medication with wild herbs or nonprescription medicines is common in Taiwan. Information regarding patterns of herbal and supplement use can help nutrition workers understand which products are most commonly used, whether the choice of supplements is related to specific health concerns, and the potential of risks or interactions. Similarly, information on non-prescription medicine use and medical conditions is useful to health professionals in understanding the self-medication practice of their patients, and is important for researchers because medical conditions may be potential confounding factors in observational studies. Thus, an understanding of the prevalence of the use of dietary supplements and non-prescription medicine is important in protecting the health of citizens.

The purpose of this study is to assess the prevalence of use of major dietary supplements and non-prescription medicines by older adults in Taiwan and to analyse their influence by anthropometric, sociodemographic and lifestyle factors.

\section{Methods}

Data of this report are from the 1999 survey of a longitudinal cohort project titled, 'Survey of Health and Living Status of the Elderly in Taiwan' (SHLSET), conducted by the former Taiwan Provincial Institute of Family Planning (TPIFP), now a part of the Bureau of Health Promotion of the Department of 
Health of Taiwan. Multipurpose surveys were conducted on the cohort since the initiation of the project in 1989 initially involving a national probability sample of 4049 men and women, 60 years or older [16]. In 1996, an additional national probability sample of 2462 men and women, 50-66 years old, were added to the remaining sample $[17,18]$. At the time of the 1999 survey, there were 4915 available respondents, the youngest being 53 years old. Interviews were conducted in respondents' homes by trained interviewers accompanied by local health workers [19]. Advance letters were sent to all $\mathrm{D}$ the survey prior to phone calls and to make arrangement for interviews. The study was carried out with ethical standards set forth in the Helsinki Declaration of 1975, and the protocol was approved by the ethics committee of TPIFP.

In the survey, each respondent was asked to indicate his/her frequency of use of each of the listed most popular dietary supplements and non-prescription medicines, or to write down the items if not listed. The dietary supplement/nonprescription medicine list included multivitamin and mineral supplements, vitamin E, calcium, fish oil, phospholipids, 'health foods', and pain-relieving, anxiety-relieving, sleepeasing and mood-lifting medicines and Chinese herbal medicine (CHM), and an open space for listing 'others'. The list was prepared based on results of preliminary studies conducted prior to the survey. For each item, the subject could choose 'use regularly' (defined as three or more times/ week), 'use occasionally or as needed' (defined as between one time/month to less than three times/week), or 'never use' during the last 12 months. The survey made no attempt to record the composition or dosage of supplements, nor the reasons for taking supplements or non-prescription medicines. If necessary, respondents were asked to show the containers of supplements or medicines to the interviewers. Interviewers would classify them accordingly or write down the brand name and composition for later classifications. In this survey, multivitamin and mineral supplements also included multivitamin supplement, but did not include vitamin B-complex supplement, which was classified as a separate category. If a supplement included two or three nutrients such as iron and vitamin C, it was doubly counted. This report analysed only the portion of data pertaining to the use of dietary supplements and non-prescription medicines in the 1999 survey of the 4440 respondents (2360 men and 2080 women) who successfully completed the questionnaire [19]. Results were statistically analysed according to gender and 10-year-age ranges with the Statistical Analyses System. Binary logistic regression analyses were performed to identify the determinants of dietary supplement and non-prescription medicine use. A probability level of $<0.05$ was designated as the level of statistical significance.

\section{Result}

\section{Use of dietary supplements}

Results of the prevalence of dietary supplement use, classified according to 10-year-age range and gender, are shown in
Table 1. Multivitamin and mineral supplements, vitamin E, calcium and fish oil were the leading dietary supplements used by older adults in Taiwan. Overall, $32.4 \%$ of men and $42.7 \%$ of women, 53 years or older, consumed at least one kind of dietary supplement, regularly or occasionally, in the last 12 months. Logistic regression analyses showed that elderly women were more likely to use multivitamin and mineral supplements $(\mathrm{OR}=2.8, P<0.001)$, vitamin $\mathrm{E}(\mathrm{OR}$ $=3.8, P<0.001)$, calcium $(\mathrm{OR}=4.3, P<0.001)$ and fish oil $(\mathrm{OR}=1.9, P<0.001)$ than men (Table 2$)$. There was an increased likelihood for older elderly to use multivitamin and mineral supplements than younger elderly (for $80+$ years elderly, $\mathrm{OR}=1.62, P<0.05$, Table 2 ), but there was a decreased likelihood for people over 80 years old to take calcium supplements $(\mathrm{OR}=0.68, P<0.05$, Table 2$)$. The level of education was a strong predictor of the use of each of the four leading dietary supplements (with the exception of one value, all $P<0.001$, Table 2 ). Frequent exercise ( $>6$ days/ week) was positively associated with increased use of all four major dietary supplements (all $P<0.001$ ). Body mass index (BMI), on the other hand, did not predict the use of these four supplements.

\section{Use of non-prescription medicine}

Female respondents had a greater likelihood of using nonprescription pain-relieving $(\mathrm{OR}=1.28, P<0.05)$, anxietyrelieving $(\mathrm{OR}=1.65, P<0.01)$ and sleep-easing medicines $(\mathrm{OR}=1.55, P<0.01)$ than their male counterparts (Table 3$)$. The use of pain-relieving medicines was highest in the 70-79year age range. For women, the use of sleep-easing medicine peaked in 70-79 years range, but for men it continued to increase after age 80. A higher level of formal education (12 years or more) was associated with a reduced use of painrelieving medicines but not with the use of anxiety-relieving or sleep-easing medicines. Overweight (BMI 25.1-30) and obese conditions (BMI > 30) predicted increased use of painrelieving medicines but not other non-prescription medicines. Elderly who exercised six or more days per week used less pain-relieving medicines $(\mathrm{OR}=0.67, P<0.001)$, but not other non-prescription medicines. No correlation was observed with the use of multivitamin and mineral supplements or vitamin $\mathrm{E}$. The use of pain-relieving $(\mathrm{OR}=1.6, P<0.01)$ and sleep-easing medicines $(\mathrm{OR}=1.74, P<0.01)$ was associated with the use of supplemental calcium. The use of pain-relieving $(\mathrm{OR}=1.60$, $P<0.01)$, anxiety-relieving $(\mathrm{OR}=2.03, P<0.01)$ and sleepeasing medicines $(\mathrm{OR}=1.65, P<0.05)$ was associated with the use of fish oil.

\section{Use of Chinese herbal medicines}

Approximately $14 \%$ of elderly men and $18 \%$ of elderly women used CHM regularly or occasionally (Table 1). The use of $\mathrm{CHM}$ reduced with increased formal education. The ORs were $0.71(P<0.05), 0.59(P<0.01)$ and $0.54(P<0.01)$ for 9,12 and $16+$ years of education, respectively. CHM use was associated with the use of calcium supplement $(\mathrm{OR}=1.37$, $P<0.05)$. No other associations were observed with the use of CHM. 
Table 1: Prevalence of dietary supplement $†$ or non-prescription medicine use among men and women 53 years or older, stratified by gender and 10-year age ranges

\begin{tabular}{|c|c|c|c|c|c|c|c|c|c|c|}
\hline \multirow[b]{2}{*}{ Parameters } & \multicolumn{5}{|c|}{ Age range - Men } & \multicolumn{5}{|c|}{ Age range - Women } \\
\hline & $\begin{array}{l}53-59 \\
(459) \ddagger\end{array}$ & $\begin{array}{c}60-69 \\
(605)\end{array}$ & $\begin{array}{l}70-79 \\
(1035)\end{array}$ & $\begin{array}{l}80+ \\
(260)\end{array}$ & $\begin{array}{c}53-80+ \\
(2360)\end{array}$ & $\begin{array}{c}53-59 \\
(440)\end{array}$ & $\begin{array}{c}60-69 \\
(548)\end{array}$ & $\begin{array}{c}70-79 \\
(727)\end{array}$ & $\begin{array}{l}80+ \\
(234)\end{array}$ & $\begin{array}{c}53-80+ \\
(2080)\end{array}$ \\
\hline \multirow{2}{*}{\multicolumn{11}{|c|}{$\%$ Respondents }} \\
\hline \multirow{2}{*}{\multicolumn{11}{|c|}{$\begin{array}{l}\text { Dietary supplements: } \\
\text { Multivitamin and mineral supplement }\end{array}$}} \\
\hline & & & & & & & & & & \\
\hline Regular use & 6.97 & 9.59 & 12.93 & $9.62 * \S$ & 10.55 & 11.97 & 11.30 & 14.41 & 14.07 & 12.98 \\
\hline Occasional use & 8.71 & 3.97 & 4.83 & $3.46^{\star}$ & 5.21 & 6.87 & 6.96 & 4.97 & 4.44 & 5.87 \\
\hline \multicolumn{11}{|l|}{ Calcium } \\
\hline Regular use & 4.79 & 6.61 & 8.49 & $5.77^{\star}$ & 6.99 & 19.29 & 12.87 & 16.58 & $10.00^{*}$ & 15.29 \\
\hline Occasional use & 5.88 & 3.64 & 3.09 & $1.15^{\star}$ & 3.56 & 8.43 & 8.70 & 5.87 & $2.22^{*}$ & 6.73 \\
\hline \multicolumn{11}{|l|}{ Vitamin E } \\
\hline Regular use & 6.75 & 6.78 & 8.40 & 6.54 & 7.46 & 13.30 & 10.09 & 9.31 & $7.04^{*}$ & 10.10 \\
\hline Occasional use & 4.36 & 2.15 & 2.41 & 1.15 & 2.58 & 4.88 & 5.04 & 3.06 & $2.59^{\star}$ & 3.94 \\
\hline \multicolumn{11}{|l|}{ Fish oil } \\
\hline Regular use & 2.18 & 4.30 & 5.41 & $2.31^{\star}$ & 4.15 & 5.10 & 3.48 & 4.59 & 5.19 & 4.47 \\
\hline Occasional use & 2.83 & 2.81 & 1.64 & $1.15^{\star}$ & 2.12 & 2.00 & 3.65 & 2.30 & 1.48 & 2.50 \\
\hline \multicolumn{11}{|l|}{ Lecithin } \\
\hline Regular use & 2.61 & 1.65 & 1.64 & 0.77 & 1.74 & 2.44 & 1.74 & 1.79 & 1.48 & 1.87 \\
\hline Occasional use & 1.09 & 0.99 & 1.35 & 0.38 & 1.10 & 0.89 & 2.43 & 1.15 & 1.11 & 1.44 \\
\hline \multicolumn{11}{|l|}{ Nutritional foods } \\
\hline Regular use & 3.49 & 3.97 & 5.02 & 2.31 & 4.15 & 5.32 & 5.04 & 5.10 & 5.56 & 5.19 \\
\hline Occasional use & 2.61 & 2.15 & 2.03 & 2.31 & 2.20 & 2.66 & 2.76 & 2.04 & 2.59 & 2.45 \\
\hline Other supplements & 7.84 & 9.42 & 9.75 & 8.84 & 9.20 & 8.65 & 11.65 & 11.10 & 10.00 & 10.57 \\
\hline \multicolumn{11}{|l|}{ Total number usedๆ } \\
\hline 1 & 15.90 & 17.52 & 18.24 & 16.92 & 17.46 & 21.95 & 20.87 & 19.52 & 20.74 & 20.58 \\
\hline 2 & 8.06 & 6.45 & 8.01 & 6.54 & 7.46 & 11.31 & 10.09 & 10.84 & 9.63 & 10.58 \\
\hline 3 & 3.49 & 3.14 & 4.34 & 2.69 & 3.69 & 7.09 & 7.13 & 6.38 & 2.96 & 6.49 \\
\hline $4+$ & 3.93 & 5.56 & 4.35 & 1.54 & 3.81 & 4.88 & 5.22 & 4.98 & 4.07 & 4.91 \\
\hline $1+$ (all) & 31.37 & 30.91 & 34.94 & 27.69 & 32.40 & 36.14 & 40.35 & 47.07 & 47.04 & 42.70 \\
\hline \multicolumn{11}{|c|}{ Non-prescription medicines } \\
\hline Pain-relieving & 9.37 & 9.55 & 13.32 & 7.31 & 10.98 & 14.63 & 15.65 & 17.47 & 17.03 & 16.29 \\
\hline Anxiety-relieving & 2.62 & 3.14 & 4.53 & $5.77^{\star}$ & 3.94 & 5.32 & 6.43 & 7.39 & 5.55 & 6.44 \\
\hline Sleep-easing & 2.61 & 3.14 & 5.98 & $7.69^{*}$ & 4.79 & 5.99 & 6.78 & 10.98 & 7.03 & 8.22 \\
\hline Mood-lifting & 3.49 & 1.98 & 1.06 & $1.92^{*}$ & 1.87 & 1.78 & 2.09 & 1.38 & $0.74^{*}$ & 1.54 \\
\hline $\mathrm{CHM}$ & 17.21 & 14.88 & 12.76 & 13.70 & 14.19 & 17.96 & 16.87 & 17.73 & 22.59 & 18.17 \\
\hline
\end{tabular}

†Most dietary supplements were USA-made such as Centrum and GNC brands. A typical dose of multivitamin and mineral supplement may include $2000 \mathrm{IU}$ of preformed vitamin A,

1500 beta-carotene, $60 \mathrm{mg}$ vitamin C, $400 \mathrm{IU}$ vitamin D, $45 \mathrm{IU}$ vitamin $\mathrm{E}, 10 \mu \mathrm{g}$ vitamin $\mathrm{K}, 1.5 \mathrm{mg}$ thiamin, $1.7 \mathrm{mg}$ riboflavin, $20 \mathrm{mg}$ niacin, $3 \mathrm{mg} \mathrm{B6,} 400 \mu \mathrm{g}$ folic acid, $25 \mu \mathrm{g}$ B12, $30 \mu \mathrm{g}$ biotin, $10 \mathrm{mg}$ pantothenic acid, $200 \mathrm{mg}$ calcium, $48 \mathrm{mg}$ phosphorus, $150 \mu \mathrm{g}$ iodine, $100 \mathrm{mg}$ magnesium, $15 \mathrm{mg}$ zinc, $20 \mu \mathrm{g}$ selenium, 2 mg copper, 2 mg manganese, $150 \mu \mathrm{g}$ chromium, $75 \mu \mathrm{g}$ molybdenum, $72 \mathrm{mg}$ choline, $80 \mathrm{mg}$ potassium, $150 \mu \mathrm{g}$ boron, $5 \mu \mathrm{g}$ nickel, $2 \mathrm{mg}$ silicon, $10 \mu \mathrm{g}$ vanadium, $250 \mu \mathrm{g}$ lutein and $300 \mu \mathrm{g}$ lycopene; a typical dose of calcium supplement usually contains 250-600 mg calcium; a typical dose of vitamin E usually contains 100-1000 IU of vitamin E; a typical dose of fish oil usually contains 1-3 g of deep marine fish body oil, and a typical dose of lecithin usually contains $1-3 \mathrm{~g}$ of soy lecithin.

†Number of observations. $\mathrm{N}$ for each age range varies slightly among variables.

$\S^{\star}$ Significant $(P<0.05)$ age-associated trend on basis of Pearson's $\chi^{2}$ test.

Ilncluding both regular and occasional uses.

$\mathrm{CHM}$, Chinese herbal medicine.

\section{Discussion}

Dietary supplements

The rate of supplement use is relatively low in Taiwan compared to that in the USA, but is comparable to many other industrialised countries. In the USA, the VITAL (Vitamin and Lifestyle) study [20], a cross-sectional survey conducted in 2000-2002, showed that $75 \%$ of participants, aged 50-75 years, used at least one dietary supplement, $57 \%$ used multivitamin and mineral supplements, $42 \%$ used vitamin E, 38\% vitamin $\mathrm{C}$ and $41 \%$ calcium. The National Health Interview Survey (NHIS), based on year 2000 data, reported that $39.8 \%$ of persons aged 65 years or older used multivitamin and mineral supplements, $22.9 \%$ used vitamin E, and $20.5 \%$ used calcium on a daily basis [1]. The prevalence of supplement use in Taiwan is comparable to that observed in the neighbouring country, Japan, where a 1999 survey showed that $28.7 \%$ used multivitamin and mineral supplements in adults aged 60 years or older [5].

Vitamin C, which is one of the most popular supplements in Western countries, is not among the most widely used ones in the current study. The reason could be because of the fact that it was not listed on the questionnaire (although there was a blank for naming 'others') since it was not among the most popular ones identified in preliminary studies. However, other studies conducted in Taiwan do show that vitamin $\mathrm{C}$ is among the most widely used supplements, at least in the general population. The NAHSIT-I survey showed that $11.7 \%$ of male and $14.2 \%$ of female subjects, 13 years old or older, used vitamin $C$ [21]. Further studies are needed to resolve this discrepancy. 
Tsai AC, Liou J-C, Chang JMC et al.

Table 2: Binary logistic regression analysis of demographic, anthropometric and physical activity parameters with use of major dietary supplements in older adults in Taiwan $(N=4440)$

\begin{tabular}{|c|c|c|c|c|c|c|c|c|c|c|c|c|c|}
\hline \multirow[b]{2}{*}{ Variables } & \multirow{2}{*}{$\begin{array}{l}\% \text { of } \\
\text { Cohort }\end{array}$} & \multicolumn{3}{|c|}{ Vitamin-mineral } & \multicolumn{3}{|c|}{ Vitamin E } & \multicolumn{3}{|c|}{ Calcium } & \multicolumn{3}{|c|}{ Fish oil } \\
\hline & & $\%$ User & OR & Range & $\%$ User & OR & Range & $\%$ User & $\mathrm{OR}$ & Range & $\%$ User & OR & Range \\
\hline $\begin{array}{l}\text { Total } \\
\text { Gender }\end{array}$ & 100 & 17.3 & & & 12.4 & & & 16.0 & & & 6.9 & & \\
\hline $\begin{array}{l}\text { Male } \\
\text { Female }\end{array}$ & $\begin{array}{l}53.2 \\
46.8\end{array}$ & $\begin{array}{l}15.8 \\
18.9\end{array}$ & $\begin{array}{l}\text { Ref } \\
2.80\end{array}$ & $2.29-3.42^{\star \star \star}$ & $\begin{array}{l}10.1 \\
14.1\end{array}$ & $\begin{array}{l}\text { Ref } \\
3.80\end{array}$ & $3.01-4.81^{\star \star \star}$ & $\begin{array}{l}10.6 \\
22.1\end{array}$ & $\begin{array}{l}\text { Ref } \\
4.32\end{array}$ & $3.51-5.32^{* \star *}$ & $\begin{array}{l}6.3 \\
7.1\end{array}$ & $\begin{array}{l}\text { Ref } \\
1.87\end{array}$ & $1.41-2.48^{\star \star \star}$ \\
\hline Age & & & & & & & & & & & & & \\
\hline $53-59$ & 20.5 & 17.3 & Ref & & 14.6 & Ref & & 19.1 & Ref & & 6.0 & Ref & \\
\hline $60-69$ & 26.6 & 16.0 & 1.06 & $0.82-1.37$ & 12.0 & 0.93 & $0.70-1.24$ & 15.9 & 0.87 & $0.68-1.12$ & 7.3 & 1.34 & $0.92-1.93$ \\
\hline 70-79 & 41.0 & 18.5 & 1.30 & $1.03-1.65^{*}$ & 11.5 & 0.98 & $0.75-1.27$ & 16.3 & 1.00 & $0.80-1.26$ & 7.0 & 1.31 & $0.92-1.86$ \\
\hline $\begin{array}{c}80+ \\
\text { Education }\end{array}$ & 11.9 & 16.0 & 1.62 & $1.16-2.27^{\star \star}$ & 8.9 & 1.08 & $0.72-1.60$ & 9.8 & 0.68 & $0.47-0.99^{*}$ & 5.3 & 1.13 & $0.67-1.93$ \\
\hline No formal & 34.2 & 9.3 & Ref & & 5.4 & Ref & & 11.7 & Ref & & 4.5 & Ref & \\
\hline 6 years & 41.8 & 15.7 & 2.59 & $2.02-3.32^{\star \star \star}$ & 10.1 & 2.71 & $2.01-3.65^{\text {***}}$ & 15.3 & 2.08 & $1.65-2.62^{\text {***}}$ & 5.8 & 1.54 & $1.08-2.20^{*}$ \\
\hline 9 years & 10.3 & 25.1 & 4.86 & $3.54-6.69^{* \star *}$ & 20.6 & 6.81 & $4.73-9.82^{\star \star \star}$ & 25.1 & 4.45 & $3.26-6.08^{* * *}$ & 11.2 & 3.17 & $2.05-4.91^{\text {*** }}$ \\
\hline 12 years & 7.5 & 32.5 & 7.99 & $5.67-11.3^{\star \star \star}$ & 29.0 & 12.34 & $8.38-18.2^{\text {*** }}$ & 21.7 & 4.12 & $2.88-5.90^{\star * \star}$ & 11.5 & 3.59 & $2.21-5.82^{\star \star \star}$ \\
\hline 16 years & 6.1 & 45.1 & 14.68 & $10.3-21.0^{\star \star \star}$ & 33.0 & 17.43 & $11.6-26.2^{\star \star \star}$ & 28.6 & 7.13 & $4.91-10.4^{\star \star \star}$ & 14.9 & 4.87 & $2.97-7.99^{\text {** }}$ \\
\hline 3Ml & & & & & & & & & & & & & \\
\hline$<19.0$ & 8.2 & 14.4 & 0.76 & $0.53-1.07$ & 8.9 & 0.80 & $0.53-1.20$ & 14.1 & 1.01 & $0.72-1.43$ & 5.2 & 0.84 & $0.50-1.41$ \\
\hline $19.1-25$ & 63.6 & 18.2 & Ref & & 12.8 & Ref & & 16.2 & Ref & & 6.6 & Ref & \\
\hline $25.1-30$ & 24.8 & 16.2 & 0.84 & $0.68-1.03$ & 12.6 & 1.00 & $0.79-1.26$ & 16.1 & 0.95 & $0.77-1.17$ & 7.1 & 1.06 & $0.79-1.42$ \\
\hline$>30$ & 3.5 & 17.0 & 0.91 & $0.57-1.45$ & 9.5 & 0.67 & $0.37-1.21$ & 21.8 & 1.19 & $0.77-1.82$ & 6.8 & 1.03 & $0.52-2.01$ \\
\hline Physical activity & & & & & & & & & & & & & \\
\hline$<1 /$ week & 41.8 & 11.4 & Ref & & 6.6 & Ref & & 12.1 & Ref & & 4.5 & Ref & \\
\hline 1-2/week & 6.8 & 21.5 & 1.72 & $1.23-2.42^{\star \star}$ & 16.6 & 2.02 & $1.37-2.97^{\star \star *}$ & 15.6 & 1.08 & $0.74-1.5$ & 9.9 & 2.03 & $1.27-3.24^{\star *}$ \\
\hline 3-5/week & 10.4 & 17.1 & 1.26 & $0.92-1.71$ & 12.6 & 1.52 & $1.07-2.20^{*}$ & 18.2 & 1.41 & $1.05-1.90^{*}$ & 4.8 & 0.98 & $0.59-1.61$ \\
\hline$>$ 6/week & 41.0 & 22.5 & 1.84 & $1.50-2.26^{\star \star \star}$ & 16.5 & 2.19 & $1.71-2.80^{\star * *}$ & 19.4 & 1.58 & $1.29-1.94^{* \star *}$ & 8.8 & 1.79 & $2.32-2.44^{\star * \star}$ \\
\hline
\end{tabular}

${ }^{*},{ }^{* *}$ and ${ }^{* * \star}$ Statistical significance at $P<0.05,0.01$ and 0.001 , respectively. BMI, body mass index. Ref, as the reference value ' 1 '.

Among other (non-vitamin non-mineral) nutritional supplements, fish oil and lecithin were most popular. Roughly the same proportion of men $(6.27 \%)$ and women $(6.97 \%)$ used fish oil, while only $2.84 \%$ of men and $3.31 \%$ of women used lecithin. As of 1999 , most other nutritional supplements such as melatonin, shark cartilage, glucosamine and chondroitin sulphate, or single item herbal supplements such as Ginkgo, Echinacea, and St. John's Wort that were popular in the US or other Western countries were not generally available in Taiwan. Unlike in the US, where dietary supplements can be produced rather freely, Taiwan is quite restrictive on their production. Manufacturing of dietary supplements or CHM generally requires government approval. As a result, foreign brands such as Centrum, GNC or their equivalents dominate the market.

Surprisingly, data on the use of dietary supplements in Taiwan are relatively incomplete or unavailable. A preliminary report of the Nutrition and Health Survey in Taiwan (NAHSIT-I) [21] indicated that $22.3 \%$ of men and $22.6 \%$ of women, aged 65 years or older, used at least one kind of dietary supplement on a regular or occasional basis. Another report [22] using data collected in 1994 on people, 25 years or older, indicated that $12.7 \%$ of males and $22.2 \%$ of females used any vitamin supplement, while $3.7 \%$ of males and $10.2 \%$ of females took calcium supplements. Although age-range differences among current and these previous studies preclude direct comparison, the use of dietary supplements appears to be on the rise. Factors contributing to this increase include improving economic conditions, media liberalisation, increasing access to international travel, widespread use of the Internet, Westernisation of lifestyle, increased product availability in consumer market, and intensified marketing in recent years.

Results of this survey indicate that elderly, who are female, more highly educated or physically active, are more likely to use multivitamin and mineral supplements, calcium, vitamin E and fish oil. Older elderly are also more likely to use multivitamin and mineral supplements. The reason for this increase with age is not exactly known but most likely related to increased prescription by care physicians and increased giving by family members, particularly children, to the elderly. On the other hand, BMI shows no association with the use of these four supplements. These findings are generally in line with results observed in the USA [9,12,23,24], Taiwan [21] and Japan [25]. Higher supplement use is also associated with higher income and healthier lifestyles in numerous studies $[1,9,12,20,26,27]$.

\section{Non-prescription and Chinese herbal medicines}

Pain-relieving, anxiety-relieving, sleep-easing and Chinese herbal medicines are the most popular non-prescription medicines used by the elderly in Taiwan. (Some CHM are prescribed by doctors specialising in Chinese medicine or by herbalists, some are private formulations, while some are ready-to-use over-the-counter items.) Women are more frequent users of nonprescription pain-relieving, anxiety-relieving and sleep-easing medicines. Elderly in the 70-79-year age range are more likely $(P<0.05)$ to use each of these non-prescription medicines and CHM than their younger (53-69 years old) counterparts. 
Table 3: Binary logistic regression analysis of the correlates of the usage of major non-prescription medicines in older adults in Taiwan $(N=4440)$

\begin{tabular}{|c|c|c|c|c|c|c|c|c|c|c|c|c|c|}
\hline \multirow[b]{2}{*}{ Variables } & \multirow{2}{*}{$\begin{array}{l}\% \text { of } \\
\text { Cohort }\end{array}$} & \multicolumn{3}{|c|}{ Pain relieving } & \multicolumn{3}{|c|}{ Anxiety relieving } & \multicolumn{3}{|c|}{ Sleep easing } & \multicolumn{3}{|c|}{ Chinese herbal medicine } \\
\hline & & $\%$ of user & OR & Range & $\%$ of user & $\mathrm{OR}$ & Range & $\%$ of user & $\mathrm{OR}$ & Range & $\%$ of user & OR & Range \\
\hline $\begin{array}{l}\text { Total } \\
\text { Gender }\end{array}$ & 100 & 13.6 & & & 5.2 & & & 6.4 & & & 16.1 & & \\
\hline Male & 53.2 & 11.1 & Ref & & 4.1 & Ref & & 4.8 & Ref & & 14.3 & Ref & \\
\hline Female & 46.8 & 16.3 & 1.28 & $1.04-1.59^{*}$ & 6.6 & 1.65 & $1.18-2.30^{\star *}$ & 8.2 & 1.55 & $1.14-2.12^{\star *}$ & 18.2 & 1.10 & $0.90-1.34$ \\
\hline Age & & & & & & & & & & & & & \\
\hline $53-59$ & 20.5 & 12.1 & Ref & & 4.0 & Ref & & 4.3 & Ref & & 17.6 & Ref & \\
\hline 60-69 & 26.6 & 12.7 & 1.07 & $0.81-1.42$ & 5.0 & 1.25 & 0.80-1.95 & 5. & 1.23 & $0.79-1.94$ & & 0.85 & $0.67-1.09$ \\
\hline $70-79$ & 41.0 & 15.3 & 1.48 & $1.14-1.92^{\star \star}$ & 5.9 & 1.52 & $1.01-2$ & 8.1 & 2.16 & $1.44-3.23^{\star \star \star}$ & 1 & 0.81 & $1.00^{*}$ \\
\hline $80+$ & 11.9 & 12.3 & 1.05 & $0.72-1.52$ & 5.8 & 1.61 & $0.93-2.78$ & 7.4 & 1.98 & $1.18-3.32^{\star \star}$ & 17.9 & 1.01 & $0.74-1.39$ \\
\hline Education & & & & & & & & & & & & & \\
\hline No formal & 34.2 & 16.2 & Ref & & 5.6 & Ref & & 7.6 & Ref & & 17.9 & Ref & \\
\hline 6 years & 41.8 & .1 & 0.88 & $0.70-1.11$ & 5.0 & 1.14 & $0.80-1.64$ & 5.4 & 0.82 & $0.59-1.14$ & 16.4 & 0.91 & $0.74-1.13$ \\
\hline 9 years & 10.3 & 1 & 0.85 & $0.59-1$ & 5.9 & 1.17 & 0.6 & 6.1 & 0.86 & 0.52 & & 0.71 & $.99^{*}$ \\
\hline 12 years & 7.5 & & 0.62 & $0.39-0.97^{*}$ & 4.1 & 0.90 & $0.46-1.76$ & 4.8 & 0.74 & $0.40-1.37$ & 8 & 0.59 & $0.39-0.87^{\star \star}$ \\
\hline 16 years & 6.1 & 8.6 & 0.54 & $0.32-0.89^{*}$ & 5.1 & 1.05 & $0.52-2.10$ & 7.5 & 1.00 & $0.54-1.86$ & 12.2 & 0.54 & $0.34-0.85^{\star \star}$ \\
\hline BMI & & & & & & & & & & & & & \\
\hline$<19.0$ & 8.2 & 14.4 & 1.13 & $0.80-1.59$ & 6.9 & 1.43 & $0.89-2.30$ & 7.8 & 1.25 & $0.81-1.93$ & 19.9 & 1.28 & $0.95-1.73$ \\
\hline $19.1-25.0$ & 63.6 & 1 & Ref & & 5.0 & Ref & & 6 & Ref & & 1 & Ref & \\
\hline $25.1-30.0$ & 24.8 & 3 & 1.24 & 1.00 & 4.9 & 1.01 & 0.7 & 5. & 0.96 & 0.7 & & 0.85 & \\
\hline $30.1+$ & 3.5 & 21.1 & 1.76 & $1.14-2.70^{*}$ & 5.4 & 1.06 & $0.50-2.23$ & 2.7 & 0.40 & $0.14-1.10$ & 13.6 & 0.74 & $0.45-1.22$ \\
\hline Physical activity & & & & & & & & & & & & & \\
\hline Little & 41.8 & 15.0 & Ref & & 5.8 & Ref & & 6.9 & Ref & & 16.5 & Ref & \\
\hline 1-2/week & 6.8 & 1 & 1.20 & $0.85-1.70$ & 5.3 & 0.95 & $0.54-1.69$ & 5.6 & 0.92 & $0.53-$ & & 1.47 & $1.06-2.03$ \\
\hline 3-5/week & 10.4 & 16.9 & 1.12 & $0.84-1.51$ & 5.2 & 0.95 & 0. & 6. & 0.90 & 0.5 & & 1.20 & .59 \\
\hline$>$ 6/week & 41.0 & 10.7 & 0.67 & $0.54-0.84^{\star \star \star}$ & 4.7 & 0.80 & $0.54-1.18$ & 6.1 & 0.88 & $0.65-1.19$ & 14.6 & 0.97 & $0.80-1.19$ \\
\hline Vitamin-minera & & & & & & & & & & & & & \\
\hline No & 82.7 & 1 & Ref & & 4.5 & Ref & & 5.7 & Ref & & & Ref & \\
\hline Yes & 17.3 & 1 & 1.15 & $0.86-1.53$ & 8.7 & 1.39 & $0.93-2.09$ & 9.8 & 1.01 & $0.69-1.49$ & 17.7 & 0.97 & $0.74-1.28$ \\
\hline Vitamin E & & & & & & & & & & & & & \\
\hline No & 88.0 & 13.2 & Ref & & 4.7 & Ref & & 5.8 & Ref & & 15 & Ref & \\
\hline Yes & 12.0 & & 1.06 & $0.76-1.47$ & 9.4 & 1.31 & $0.83-2.07$ & 11.3 & 1.24 & $0.81-1.90$ & 20.5 & 1.32 & $0.98-1.79$ \\
\hline Calcium & & & & & & & & & & & & & \\
\hline No & 84.0 & 12.8 & Ref & & 4.6 & Ref & & 5.4 & Ref & & 15 & Ref & \\
\hline $\begin{array}{l}\text { Yes } \\
\text { sh oil }\end{array}$ & 16.0 & & 1.60 & $1.12-2.28^{\star \star}$ & 8.7 & 1.15 & $0.76-1.72$ & 12.0 & 1.74 & $1.21-2.51^{\star *}$ & 22.0 & 1.37 & $1.06-1.78^{*}$ \\
\hline No & 93.3 & 13.0 & Ref & & 4.8 & Ref & & 5.9 & Ref & & 15.7 & Ref & \\
\hline Yes & 6.7 & 21.6 & 1.60 & $1.12-2.28^{\star \star}$ & 12.2 & 2.03 & $1.27-3.23^{\star \star}$ & 14.2 & 1.65 & $1.05-2.59^{*}$ & 21.3 & 1.02 & $0.71-1.46$ \\
\hline
\end{tabular}

${ }^{*},{ }^{* \star}$ and ${ }^{\star \star \star}$ statistical significance at $P<0.05,0.01$ and 0.001 , respectively. BMl, body mass index; $0 \mathrm{R}$, odds ratio. Ref, as the reference value ' 1 '.

Elderly over 80 years old also have increased $(P<0.01)$ use of sleeping-easing medicine but not the others. The level of formal education is also associated with the use of some but not all non-prescription medicines. Those who have had high school or higher levels of education are less likely to use non-prescription pain-relieving medicine or CHM. It is probable that the reduced use of pain-relieving medicine and CHM by more highly educated elderly is because they seek medical help mostly from the professionals and practise less self-medication. Overweight or obese elderly use non-prescription pain-relieving medicines more frequently but physically active elderly use less of these medicines. However, it is not possible to determine the casual relationship in a cross-sectional study. Those elderly who need to use pain-relieving medicines might not be in a condition to perform physical exercise. Those who can exercise might have better health and therefore have less need for taking pain-relieving medicines. Elderly who take calcium supplements are more likely to also use non-prescription sleepeasing medicines or CHM. The reasons for these associations are not apparent. It is interesting to note that use of fish oil is associated with increased use of non-prescription pain-relieving, anxiety-relieving and sleep-easing drugs. These associations could be because of the belief by some that consumption of fish oil may benefit such health conditions. However, the real reasons remain to be determined.

Mood-lifting products are more popular among younger males. These products usually contain stimulants such as caffeine and high levels of B vitamins. They are usually oral tonics to be consumed by people who wish to work long hours or are under physical stress. Younger male respondents (53-59 years) are frequent users and the frequency of use decreased after retirement age.

It is well recognised that there are significant differences between the East and the West in the way herbs are used for health therapies. In traditional Chinese medicine, herbs are mainly used as ingredients of formulations, whereas in the West herbs are most often used as single-item therapies. In the USA, the NHIS 2000 survey showed that $14.5 \%$ of adults reported using herbal remedies and single-ingredient products. Echinacea, ginkgo biloba and garlic preparations were the 
leading items [1]. In Japan, a 2001 survey showed that 17.2\% of adults used herbs and over-the-counter Kampo (Japanese $\mathrm{CHM}$ ), and $10 \%$ used ethical Kampo (Kampo prescribed by medical doctors) among adults, 60-79 years old, in the past 12 months [26].

It appears that the popularity of CHM among Chinese populations across different countries remains relatively high, despite the dominant use of modern Western medicines. A recent report showed that $25.3 \%$ of Chinese in Singapore used CHM over the last year [28]. In Taiwan, the current study showed that $14 \%$ of elderly men and $18 \%$ of elderly women used CHM.

\section{General discussion}

We must keep in mind that the lack of consistency in the definition of many dietary supplements, and the differences in the criteria and classification among various surveys highlight the difficulty in making direct comparison among studies. In the current case, the lack of a detailed dietary record further makes it more difficult to relate the frequency of supplement use to the total intake of each nutrient. Furthermore, in a cross-sectional study, results can suggest associations but, in most cases, do not allow inference of causal relationships.

It would be of interest to analyse the motivations for taking dietary supplements by the elderly in Taiwan. Unfortunately, the questionnaire of the SHLSET survey did not include such questions. People generally take dietary supplements for curative, corrective or health-optimising purposes. Studies conducted in younger subjects in Taiwan showed that approximately $20 \%$ of users took multivitamin and mineral supplements according to doctor's prescriptions. Others are for self-treatment of an existing health condition (especially using Chinese herbs) or for correcting a low intake of a nutrient (such as taking calcium supplement), or for optimising one's overall health condition (such as taking vitamin $\mathrm{E}$ or multivitamin and mineral supplements) [22]. For the elderly, based on our observations, doctor's recommendations appear to be a major contributing factor, particularly pertaining to the use of calcium and multivitamin and mineral supplements. The Taiwanese diet is known to be relatively deficient in calcium because the majority of people do not consume milk or cheese regularly and there are relatively few other calcium-rich foods. Government health agencies encourage citizens to consume calcium-rich foods or to take calcium supplements. For other supplements, it appears that most users take these products based on their own choice after learning the potential health benefits from the media. At the present time, the government in Taiwan does not take a stand on the use of dietary supplements (except calcium). Rather, it tries mainly to play a safeguarding role to ensure the safety of these products.

The widespread use of dietary supplements and non-prescribed herbal products raises some concern about their safety and interaction with nutrients. Self-medication with $\mathrm{CHM}$ has been reported to cause health problems [29-31]. There have been reports of adulterations of CHM with toxic heavy metals and undeclared chemicals such as steroids and antibiotics. Like in most other countries, physicians do not usually inquire about and few patients inform their care physicians about the use of alternative or herbal medicines [32,33]. Poly-pharmacy with modern prescription drugs for chronic disorders is also common in elderly persons. Drug-nutrient or nutrient-nutrient interactions are common. Thus, the potential adverse effects from high dosage of supplemental nutrients or herbs are of great clinical concern. Obviously, there is a clear need to increase public awareness of the potential risk of supplement use or self-medication.

\section{Acknowledgements}

This study was funded by the former Taiwan Provincial Institute of Family Planning, now a component of the Bureau of Health Promotion of the Department of Health, Taiwan. The study was conducted by the Institute (TPIFP) in collaboration with the School of Public Health of the University of Michigan, Ann Arbor, Michigan, USA. The authors wish to thank the field interviewers and local health workers who diligently completed the personal interviews. The authors also wish to thank Dr Chien Sze for her valuable input in viewing this manuscript.

\section{Key Points}

- Dietary supplement use appears on the rise among older adults in Taiwan in recent years.

- The major dietary supplements used by elderly Taiwanese are multivitamin and mineral supplements, vitamin E, calcium and fish oil.

- Approximately $32 \%$ of men and $43 \%$ of women used at least one type of dietary supplement in the past 12 months.

- Higher rates of dietary supplement use were observed in elderly who are female, more educated and physically active.

\section{References}

1 Millen AE, Dodd KW, Subar AF. Use of vitamin, mineral, nonvitamin, and nonmineral supplements in the United States: the 1987, 1992, and 2000 National Health Interview Survey Results. Journal of the American Dietetic Association 2004; 104: 942-950.

2 Messerer M, Johansson SE, Wolk A. Use of dietary supplements and natural remedies increased drastically during the 1990s. Journal of Internal Medicine 2001; 250: 160-166.

3 Harris P, Rees R. The prevalence of complementary and alternative medicine use among the general population: a systematic review of the literature. Complementary Therapies in Medicine 2000; 8: 88-96.

4 Radimer K, Bindewald B, Hughes J et al. Dietary supplement use by US adults: data from the national health and nutrition examination survey, 1999-2000. American Journal of Epidemiology 2004; 160: 339-349.

5 Flaherty JH, Takahashi R, Teoh J et al. Use of alternative therapies in older outpatients in the United States and Japan: prevalence, reporting patterns, and perceived effectiveness. Journal of Gerontology: Medical Sciences 2001; 56A: M650-M655.

6 Radimer KL, Suber AF, Thompson FE. Nonvitamin, nonmineral dietary 
supplements: issues and findings from NHANES III. Journal of the American Dietetic Association 2000; 100: 447-454.

7 Kelly JP, Kaufman DW, Kelly K, Rosenberg L, Anderson TE, Mitchell AA. Recent trends in use of herbal and other natural products. Archives of Internal Medicine 2005; 165: 281-286.

8 Ervin RB, Kennedy-Stephenson J. Mineral intakes of elderly adult supplement and non-supplement users in the Third National Health and Nutrition Examination Survey. Journal of Nutrition 2002; 132: 3422-3427.

9 Neuhouser ML, Kristal AR, Patterson RE et al. Dietary supplement use in the Prostate Cancer Prevention Trial: implications for prevention trials. Nutrition and Cancer 2003; 39: 12-18.

10 Holmquist C, Larsson S, Wolk A, de Faire U. Multivitamin supplements are inversely associated with risk of myocardial infarction in men and women - Stockholm Heart Epidemiology Program (SHEEP). Journal of Nutrition 2003; 133: 2650-2654.

11 Rimm EB, Willet WC, Hu FB et al. Folate and vitamin B6 from diet and supplements in relation to risk of coronary heart disease among women. Journal of the American Dietetic Association 1998; 279: 359-264.

12 Gunther S, Patterson RE, Kristal AR et al. Demographic and health-related correlates of herbal and specialty supplement use. Journal of the American Dietetic Association 2004; 104: 27-34.

13 Stupay S, Sivertsen L. Herbal and nutritional supplement use in the elderly. Nurse Practitioner 2000; 25: 56-58.

14 Smolinske SC. Dietary supplement-drug interactions. Journal of the American Medical Women's Association 1999: 54: 191-192.

15 Sorensen JM. Herb-drug, food-drug, nutrient-drug, and drug-drug interactions: mechanisms involved and their medical implications. Journal of Alternative and Complementary Medicine 2002; 8: 293-308.

16 Taiwan Provincial Institute of Family Planning. 1989 Survey of health and living status of the elderly in Taiwan: Questionnaire and survey design. Comparative study of the elderly in four Asian countries. Research report No. 1/December 1989

17 Chang MC, Hermalin Al. 1996 Survey of health and living status of the middle aged and elderly in Taiwan. (A) Survey of those over 50-66 years of age. Elderly in Asia research report No. 98-49, April 1998.

18 Chang MC, Hermalin Al. 1996 Survey of health and living status of the middle aged and elderly in Taiwan. (B) Survey of those over 67 years of age. Elderly in Asia research report No. 98-50, April 1998.

19 Tsai AC, Chang JMC, Lin $\mathrm{H}$ et al. Assessment of nutritional risk of $>$ 53year-old men and women in Taiwan. Public Health Nutrition 2004; 7 : 69-76.
20 Satia-About AJ, Kristal AR, Patterson RE et al. Dietary supplement use and medical conditions. The VITAL Study. American Journal of Preventive Medicine 2003; 24: 43-51.

21 Department of Health (Taiwan). Report of the 'First Nutrition and Health Survey in Taiwan', 1992-97 (NAHSIT-I). Taipei, Taiwan: Department of Health, Executive Yuen, 1998

22 Chang $\mathrm{CH}$, Chiang TL. Vitamin/calcium supplement use in Taiwan: findings from the 1994 National Health Interview Survey. Kaohsiung Journal of Medical Sciences 2002; 18: 171-181.

23 Messerer M, Johansson SE, Wolk A. Sociodemographic and health behavior factors among dietary supplement and natural remedy users. European Journal of Clinical Nutrition 2001; 55): 1104-1110.

24 Jasti S, Siega-Riz AM, Bentley ME. Dietary supplement use in the context of health disparities: cultural, ethnic and demographic determinants of use. Journal of Nutrition 2003; 133: 2010S-2013S

25 Ishihara J, Sobue T, Yamamoto S, Sasaki S, Tsugane S; JPH Study Group. Demographics, lifestyles, health characteristics, and dietary intake among dietary supplement users in Japan. International Journal of Epidemiology 2003; 32: 553-555.

26 Yamashita H, Tsukayama H, Sugishita C. Popularity of complementary and alternative medicine in Japan: a telephone survey. Complementary Therapies in Medicine 2002; 10: 84-93.

27 Kirk SF, Cade JE, Barrett JH, Conner M. Diet and lifestyle characteristics associated with dietary supplement use in women. Public Health Nutrition 1999: 2: 69-73

$28 \mathrm{Ng}$ TP, Tan CH, Hua EH. The use of Chinese herbal medicines and their correlates in Chinese older adults: the Singapore Chinese Longitudinal Aging Study. Age and Ageing 2004; 33: 135-142.

29 Chang $\mathrm{CH}$, Wang YM, Yang AH, Chiang SS. Rapidly progressive interstitia renal fibrosis associated with Chinese herbal medications. American Journal of Nephrology 2001; 21: 441-448.

30 Tanaka A, Nishida R, Yoshida T et al. Outbreak of Chinese herb nephropathy in Japan: are there any differences from Belgium? Internal Medicine 2001; 40: 296-300.

31 Chan TY, Chan JC, Tomlinson B, Critchley JA. Chinese herbal medicines revisited: a Hong Kong perspective. Lancet 1993; 342: 1532-1534.

32 Eisenberg DM, Davis RB, Ettner SL et al. Trends in alternative medicine use in the United States, 1990-97. Result of a follow-up national survey. Journal of American Medical Association 1998; 280: 1569-1575.

33 Shaw D, Leon C, Murray V, Volans F. Patients' use of complementary medicine (letter). Lancet 1998; 352: 408. 